\title{
Anxiety, Depression, Stress and the Heart: When Measurement Matters
}

\author{
Cristiano Tschiedel Belem da Silva ${ }^{\circledR}$ and Rafaela Doebber Escobar ${ }^{\circledR}$ \\ Universidade Federal do Rio Grande do Sul,' Porto Alegre, RS - Brazil \\ Universidade do Vale do Rio dos Sinos, ${ }^{2}$ São Leopoldo, RS - Brazil
}

Editorial related to the article: Validation of the Brazilian Version of the Screening Tool for Psychosocial Distress (Stop-D) for Cardiac Patients

The interplay between cardiovascular symptoms and stress has been a matter of concern for centuries. The first known scientific description of panic disorder, for example, which would nowadays be considered a mixture of panic disorder itself, post-traumatic-stress disorder, cardiac arrhythmia and congestive heart failure, took place in a cohort of three hundred patients of a military hospital in Philadelphia (USA). The name of the new diagnostic entity that followed ("irritable heart syndrome" or simply "Da Costa Syndrome," named after the author of the original description) is rather an indicator of the historical overlap between cardiovascular diseases (CVD) and psychosocial conditions than properly an example of scientific rigor in describing the discovery of a new medical syndrome. ${ }^{1}$

About sixteen years after the description of the irritable heart syndrome, Augustus Waller, a British physiologist, published the first human electrocardiogram (EKG) applying a capillary electrometer and placing electrodes in the chest and back of an individual. More than half a century after Waller's publication, the 12-Lead EKG - as we know it now - was standardized by the American Heart Association. ${ }^{2}$ Since then, EKG is the most important test for the interpretation of cardiac rhythm and for the detection of myocardial ischemia. In sharp contrast with diagnostic developments in Cardiology and despite a whole new era in neuroimaging, no single physiological marker has emerged to help detecting anxiety disorders or depression in Psychiatry. ${ }^{3}$ Nonetheless, the degree to which mental disorders are realized within the brain or in the rest of the body is still unclear.

\section{Keywords}

Cardiovascular Diseases; Life Style; Stress, Psychological; Anxiety; Depression; Imaging, Diagnosis.
In real-life psychiatric clinical settings, the gold standard of diagnosis is still the judgement of an experienced clinician. Structured psychometric measures with adequate diagnostic properties do exist and are especially useful to quantify clinical response in randomized controlled trials. One particularly worrisome issue for both psychiatrists and cardiologists is that two of the most popular psychometric scales used in clinical trials poorly discriminate somatic and psychological symptoms of anxiety and depression.

In the last decades, much has been published on the association between emotional symptoms and CVD. Biological plausibility for the association is sound and research on the subject seem fairly consistent. However, one of the main criticisms to such studies is that higher scores in psychometric scales commonly used to assess symptoms of anxiety, stress or depression in patients with CVD might be a reflection of underlying acute cardiological conditions. Indeed, fatigue, sleep disturbances and appetite changes, among other symptoms, can substantially overlap in subjects with cardiovascular diseases combined with mood and anxiety disorders. Accordingly, a recent study showed that post-myocardial infarction (MI) patients with major depressive disorder (MDD) presented a three-point higher average of somatic symptoms of depression than matched controls with MDD, but without a history of MI. ${ }^{4}$

In the current issue of the International Journal of Cardiovascular Sciences, Gontijo and colleagues report the validation process of the Screening Tool for Psychosocial Distress (STOP-D) for the Brazilian population. ${ }^{5}$ Originally developed as an alternative to assess depression, anxiety, stress, anger and low social support in a single and brief 5-item instrument, STOP-D had demonstrated adequate psychometric properties

Mailing Address: Cristiano Tschiedel Belem da Silva

R. Ramiro Barcelos, 2400. Postal Code: 90035-003, Santa Cecilia, Porto Alegre - RS, Brazil.

E-mail: cristianotbs@hotmail.com

DOI: 10.5935/2359-4802.20190082 
in three outpatients cardiac clinics from Canada. ${ }^{6}$ Its validation in a Brazilian sample of cardiological patients is an important step towards the implementation of routine psychological evaluation in a poor country such as Brazil. Its widespread availability as a free instrument, brief application period and simplicity of assessment (self-report) will hopefully support the operationalization of psychological evaluation both in daily clinical practice and research studies in cardiological samples, both of which have been somewhat set aside in our country, despite worldwide trends in the opposite direction.

Remarkably, amongst the STOP-D features is the absence of somatic symptoms of stress, anxiety and depression within its items. This can be a valuable characteristic when dealing with patients with overlapping symptoms of cardiological conditions and mental disorders, but can also contribute for overlooking patients also in need of adequate psychosocial management. With this in mind, the tradeoff between assessing psychosocial issues in cardiological patients with low costs and widespread availability and possibly overlooking somatic symptoms of anxiety and depression is likely highly beneficial. Considering the low sensibility of general practitioners to detect certain kinds of associated mental disorders ${ }^{7}$ and current efforts to better elucidate health factors associated with CVD in order to reduce mortality rates, ${ }^{8}$ the study by Gontijo and colleagues will certainly find a fertile ground in our practice.

\section{References}

1. da Costa JM. On irritable heart: a clinical study of a form of functional cardiac disorder and its consequences. Am J M Sc. 1871;61(121):17-52.

2. AlGhatrif M, Lindsay J. A brief review: history to understand fundamentals of electrocardiography. J Community Hosp Intern Med Perspect. 2012;2(1):14383.

3. Insel T, Cuthbert B, Garvey M, Heinssen R, Pine DS, Quinn K, et al. Research domain criteria (RDoC): Toward a new classification framework for research on mental disorders. Am J Psychiatry. 2010;167(7):748-51.

4. Delisle VC, Beck AT, Ziegelstein RC, Thombs BD. Symptoms of heart disease or its treatment may increase Beck Depression Inventory Scores in hospitalized post-myocardial infarction patients. J Psychosom Res. 2012;73(3):157-62.

5. Gontijo IBR, Souza JR, Barbosa DF, Rassi S. Validation of the Brazilian Version of the Screening Tool for Psychosocial Distress (Stop-D) for Cardiac Patients. Int J Cardiovasc Sci. 2019;32(5):438-446.

6. Young $Q$, Ignaszewski A. Brief screen to identify 5 of the most common forms of psychosocial distress in cardiac patients validation of the screening tool for psychological distress. J Cardiovasc Nurs. 2007; 22(6):525-34.

7. Fernández A, Rubio-Valera M, Bellón JA, Pinto-Meza A, Luciano JV, Mendive JM, et al. Recognition of anxiety disorders by the general practitioner: results from the DASMAP Study. Gen Hosp Psychiatry. 2012;34(3):227-33

8. Lloyd-Jones DM, Hong Y, Labarthe D, Mozaffarian D, Appel LJ, Van Horn L, et al. Defining and setting national goals for cardiovascular health promotion and disease reduction: the American Heart Association's strategic Impact Goal through 2020 and beyond. Circulation. 2010;121(4):586-613. 